

https://doi.org/10.31533/pubvet.v13n12a465.1-4

\title{
Avaliação endoparasiticida das moléculas Moxidectina e Ivermectina contra nematódeos gastrintestinais de ovinos naturalmente infectados
}

\author{
Andressa Duarte Lorga ${ }^{10 *}$, Laura Muniz Arruda Pereira ${ }^{2} \bullet$, Luciana Maffini Heller ${ }^{3} \bullet$, Julio \\ Sylvio Dias Bortolato ${ }^{4}$, Isabelle Smaniotto Compagnoni ${ }^{5}{ }^{\circ}$, Antonio Campanha Martinez ${ }^{6}$, \\ Cláudio Alessandro Massamitsu Sakamoto ${ }^{7} 0$ \\ ${ }^{1}$ Medica veterinária, Residente em clínica médica e cirúrgica de grandes animais da Universidade Federal do Paraná, Curitiba, Paraná, Brasil \\ ${ }^{2}$ Médica veterinária pelo Centro de Ciências Agroveterinárias da Universidade do Estado de Santa Catarina, Lages, Santa Catarina, Brasil \\ ${ }^{3}$ Medica veterinária, Mestranda pelo programa de Pós-Graduação em Ciência Animal da Universidade federal de Goiás, Goiânia, Goiás, Brasil. \\ ${ }^{4}$ Médico veterinário pela Universidade Estadual de Maringá, Departamento de medicina veterinária, Umuarama, Paraná, Brasil. \\ ${ }_{5}^{5}$ Acadêmica da Universidade Estadual de Maringá, Departamento de medicina veterinária, Umuarama, Paraná, Brasil. \\ ${ }^{6}$ Professor Doutor adjunto da Universidade Estadual de Maringá, Departamento de medicina veterinária, Umuarama, Paraná, Brasil. \\ ${ }^{7}$ Professor Doutor adjunto da Universidade Federal Fluminense, Departamento de medicina veterinária, Niteroi, Rio de Janeiro, Brasil. \\ *Autor para correspondência,E-mail: lalorga2@hotmail.com
}

\begin{abstract}
Resumo. As helmintoses gastrintestinais em ovinos são o principal fator que interfere na produção e criação destes animais e se não tratada de forma adequada, pode levar ao desenvolvimento de resistência, resultando em prejuízos ao produtor. Deste modo, o trabalho tem por objetivo, determinar a atividade anti-helmíntica das formulações contendo moxidectina e ivermectina em ovinos naturalmente infectados por nematódeos gastrintestinais de propriedades rurais pertencentes à região de Umuarama-PR, através da realização de testes de redução na contagem de ovos por grama de fezes (OPG). Em duas propriedades avaliadas, ambas as formulações demonstraram ineficiência na atividade antihelmíntica em ovinos.
\end{abstract}

Palavras chave: Haemonchus, resistência anti-helmíntica, manejo, lactonas macrocíclicas

\section{Endoparasiticide evaluation of moxidectin and ivermectin molecules against gastrointestinal nematodes in naturally infected ovine}

\begin{abstract}
Gastrointestinal helminths in sheep are the main factor that interferes in the production and rearing of these animals and if not treated properly, can lead to the development of resistance, resulting in losses to the producer. In this way, the objective of this work is to determine the anthelmintic activity formulations containing moxidectin and ivermectin in sheep naturally infected by gastrointestinal nematodes belonging in Umuarama-PR region, by performing egg count reduction tests by gram of feces (OPG). In two evaluated proprieties tested, the two formulations demonstrated inefficiency in anthelmintic on sheep activity.
\end{abstract}

Keywords: Haemonchus, anthelmintic resistance, management, macrocyclic lactones

\section{Evaluación de endoparasiticidas de las moléculas Moxidectina e Ivermectina contra nematodos gastrointestinales de ovinos infectadas naturalmente}

Resumen. Los helmintos gastrointestinales en ovinos son el principal factor que interfiere en la producción y cría de estos animales y si no se tratan adecuadamente, pueden conducir al desarrollo de resistencia, resultando en daños al productor. Así, el objetivo de este trabajo es determinar la actividad antihelmíntica de formulaciones que contienen moxidectina e 
ivermectina en ovinos infectadas naturalmente por nematodos gastrointestinales de propiedades rurales pertenecientes a la región de Umuarama-PR, mediante la realización de pruebas de reducción en el recuento de óvulos por gr de heces (OPG). En dos propiedades evaluadas, ambas formulaciones demostraron ineficiencia en la actividad antihelmíntica en ovinos.

Palabras clave: Hemeniquía, resistencia antihelmintina, gestión, lactonas macrocíclicas

\section{Introdução}

A ovinocultura tem assumido importante papel na economia brasileira, atingindo o efetivo aproximado de 18 milhões de cabeças no Brasil. Associa-se esse desenvolvimento a possibilidade de utilização de seus produtos e subprodutos, pelo crescimento populacional e consequente busca por atividades agropecuárias menos onerosas e de rápido ciclo de produção (ANUALPEC, 2019; Barchet et al., 2011). Entretanto, há alguns fatores limitantes na produção de ovinos e, dentre eles, as helmintoses gastrintestinais tomam destaque, pois são reconhecidamente as principais causas de perdas na produtividade pecuária (Molento, 2004; Ramos et al., 2004), influenciam na conversão alimentar, produção de lã, desempenho reprodutivo, qualidade da carcaça, além de poder levar o animal a óbito (Amarante, 2009; Amarante, 2015; Lopes et al., 2013).

As estratégias para controlar os nematódeos gastrintestinais têm, tradicionalmente, envolvidas o uso de antihelmínticos em intervalos de 30 a 45 dias, ou no pico de transmissão das larvas, que se dá no verão (Roque et al., 1999).

Dentre os antiparasitários mais utilizados, está o grupo das lactonas macrocíclicas, anti-parasitários de amplo espectro com alta eficácia contra nematódeos (Mckellar, 1994). Deste grupo, destacam-se a moxidectina, classificada como uma milbemicina, e a ivermectina, classificada como uma avermectina. Ambas possuem como mecanismo de ação a intervenção da transmissão dos impulsos nervosos, causando paralisia no parasito (Nova et al., 2014).

Diante do exposto, o presente trabalho objetivou avaliar a capacidade endoparasiticida das moléculas moxidectina e ivermectina, que são rotineiramente utilizadas na ovinocultura, verificando por meio de testes de eficácia a viabilidade de utilizá-las.

\section{Material e métodos}

Foram selecionadas duas propriedades da região de Umuarama-PR, as quais apresentam estrutura para o manejo e pesagem dos ovinos, sendo que estes não haviam recebido tratamento anti-helmíntico há pelo menos, quarenta e cinco dias. Em cada propriedade, foram selecionados vinte quatro ovinos (oito por grupo experimental), naturalmente infectados por helmintos gastrintestinais, com contagens de ovos por grama de fezes (OPG) superiores a 300, segundo a técnica de Gordon \& Whitlock (1939).

Os animais foram dispostos em três grupos, com oito animais cada e distribuídos de forma aleatória de acordo com a contagem de OPG, sendo o Grupo I composto por animais que não receberam nenhum tratamento (controle), Grupo II, onde os animais foram submetidos ao tratamento com Moxidectina $1 \%$ $(0,2 \mathrm{mg} / \mathrm{kg}$ via subcutâneo) e o Grupo III, ao tratamento com Ivermectina $0,08 \%$ ( $0,2 \mathrm{mg} / \mathrm{Kg}$ via oral).

$\mathrm{O}$ dia do tratamento foi denominado dia zero (D0). As contagens de OPG realizaram-se, em todos os ovinos, nos dias -1 pré-tratamento, dia 7 e 14 dias pós-tratamento.

A partir dos resultados coproparasitológicos obtidos, foram calculados os percentuais de redução de OPG e eficácia do fármaco. O cálculo de redução de OPG foi realizado pelo teste, "Fecal Egg-count Reduction Test" (Teste de redução de ovos por grama de fezes-OPG), que consiste em comparar a redução de OPG num grupo de animais tratados com um anti-helmíntico com o de OPG de um grupo controle, não tratado, mantido nas mesmas condições (Vizard \& Wallace, 1987). Utilizou-se a fórmula: $\mathrm{R}=100(1-\mathrm{T} / \mathrm{C})$, onde $\mathrm{R}=$ redução calculada no OPG; $\mathrm{T}=$ média geométrica dos OPGs $(\mathrm{OPG}+10)$ dos animais tratados; $\mathrm{C}=$ média geométrica dos $\mathrm{OPGs}(\mathrm{OPG}+10)$ dos animais não tratados. $\mathrm{E}$ calculou-se a eficácia terapêutica, pela fórmula: Percentual de eficácia = (Média de OPG do Grupo Controle no dia X - Média de OPG do Grupo Tratamento no dia X) / Média de OPG do Grupo Controle no dia X (Wood 
et al., 1995). Realizou-se o cálculo nos dias pós-tratamento (dias 7 e 14 pós-tratamento). A partir da obtenção dos resultados, avaliou-se a viabilidade de utilizar os fármacos.

\section{Resultados e discussão}

A partir da interpretação dos resultados das porcentagens de eficácia e redução de OPG, notou-se a existência de resistência aos endoparasiticidas avaliados nas propriedades em questão. Segundo Thomaz-Soccol et al. (2004) a resistência anti-helmíntica das moléculas Ivermectina e Moxidectina no rebanho ovino no Paraná é de $78,6 \%$ e $23,6 \%$ respectivamente, corroborando com a pesquisa, que evidencia uma maior resistência ao fármaco Ivermectina em relação à Moxidectina. A resistência demonstrada no estado do Paraná, também é apontada no estado de Santa Catarina, onde $77 \%$ das propriedades apresentam resistência a Ivermectina (Ramos et al., 2002).

Nos dias pós-tratamento (D7 e D14), ambos os fármacos não apresentaram eficácia e redução do OPG significativas. Porém, na propriedade I, a Moxidectina apresentou uma eficácia maior se comparada a Ivermectina, pois apresentou índices de 69,10\% (D7) e 67,46\% (D14). A média da contagem de ovos por gramas de fezes (Tabela 1) demonstra essa eficácia quando compara a molécula Ivermectina.

Tabela 1. Média da contagem de ovos por grama de fezes (OPG) dos grupos Controle, tratado com Ivermectina e tratado com Moxidectina nos dias experimentais (D0, D7 e D14) da Propriedade I

\begin{tabular}{lccc}
\hline Grupos & D0 & D7 & D14 \\
\hline Controle & 3443,75 & 3721,43 & 2535,71 \\
Ivermectina & 4762,50 & 2325,00 & 3725,00 \\
Moxidectina & 4150,00 & 1150,00 & 825,00 \\
\hline
\end{tabular}

Na propriedade II, apenas a Moxidectina apresentou eficácia de 11,43\% em D7 (Tabela 2). Percentual considerado baixo, pois segundo Barnes et al. (1995) para uma formulação ser classificada como eficaz, deve apresentar eficácia mínima de 80\%.

Tabela 2. Percentual de redução de OPG nos dias após tratamento (D7 e D14) da Propriedade II.

\begin{tabular}{lcc}
\hline Grupos & $\mathrm{D} 7$ & $\mathrm{D} 14$ \\
\hline Controle & $0,00 \%$ & $26,37 \%$ \\
Ivermectina & $51,18 \%$ & $21,78 \%$ \\
Moxidectina & $72,29 \%$ & $80,12 \%$ \\
\hline
\end{tabular}

Os fármacos pesquisados não apresentaram eficácia significativa nos rebanhos testados, demonstrando a presença de resistência aos endoparasiticidas e possíveis erros de manejo, o que em conjunto trazem falhas na efetividade da vermifugação e consequentes danos aos animais. Diante dos resultados, visualizasse a necessidade de quantificar a eficácia dos endoparasiticidas mais utilizados, pois esse monitoramento permite avaliar os fármacos e suas possíveis resistências, o que devido ao uso frequente e em doses erradas acarretam o desenvolvimento de parasitos resistentes aos antiparasitários (Sakamoto et al., 2013).

\section{Conclusão}

Os resultados não foram satisfatórios, demonstrando a presença de resistência às moléculas testadas, evidenciando a necessidade de pesquisar qual o melhor antiparasitário para as propriedades em questão. Mais estudos complementares são necessários para evidenciar a situação da resistência anti-helmíntica em ovinos na região de Umuarama-PR.

\section{Referências bibliográficas}

Amarante, A. F. T. (2009). Nematoides gastrintestinais em ovinos. Doenças parasitárias de caprinos e ovinos: epidemiologia e controle. Brasília: Embrapa Informação Tecnológica, Brasília.

Amarante, A. F. T. (2015). Os parasitos de ovinos. São Paulo, Brasil: Editora da UNESP.

ANUALPEC. (2019). Anuário da Pecuária Brasileira (20th ed. Vol. 1). São Paulo, São Paulo, Brasil: Instituto FNP. 
Barchet, I., Mognon, B. A. C. \& Siluk, J. C. M. (2011). A dinâmica e o panorama da cadeia produtiva de ovinos: uma análise para identificar novas possibilidades. Paper presented at the Congresso Brasileiro de Engenharia de Produção, Ponta Grossa.

Barnes, E. H., Dobson, R. J. \& Barger, I. A. (1995). Worm control and anthelmintic resistance: adventures with a model. Parasitology Today, 1156-63.

Gordon, H. M. \& Whitlock, H. V. (1939). A new technique for counting nematode eggs in sheep faeces. Journal of the Council for Scientific and Industrial Research, 12(1):50-52.

Lopes, J., Sanches, J. M., Braga, R. M. \& Melo, D. R. (2013). Avaliação de diferentes princípios ativos no controle de helmintos gastrintestinais em rebanho ovino na região do Taiano - Roraima. Revista Brasileira de Ensino de Ciências Agrárias, 185-103.

Mckellar, Q. A. (1994). Chemoterapy and delivery systems-helminths. Veterinary Parasitology, 54249-258.

Molento, M. B. (2004). Resistência de helmintos em ovinos e caprinos. Revista Brasileira de Parasitologia, 138286.

Nova, L. E. V., Costa, M. E., Melo, P. G. C. F., Cunha Filho, L. F. C., Junior, F. A. B., Silva, L. C. \& Bogado, A. L. G. (2014). Resistência de nematoides aos anti-helmínticos nitroxinil $34 \%$ e ivermectina $1 \%$ em rebanho ovino no município de São João do Ivaí, Paraná. Revista Brasileira de Higiene e Sanidade Animal, 8(1):159171.

Ramos, V., Bellato, C. I., Souza, A. P., Avila, V. S., Coutinho, G. C. \& Dalagnoll, C. A. (2004). Epidemiologia das helmintoses gastrintestinais de ovinos no Planalto Catarinense. Ciência Rural, 34(6):1889-1895.

Ramos, C. I., Bellato, V., Ávila, V. S., Coutinho, G. C. \& Souza, A. P. (2002). Resistência de parasitos gastrintestinais de ovinos a alguns anti-helmínticos no Estado de Santa Catarina, Brasil. Ciência Rural, 32(3):473-477.

Roque, A. P., Osório, J. C. S., Jardim, P. O., Oliveira, N. M. \& Osório, M. T. (1999). Produção de carne em ovinos de cinco genótipos. 6. Desenvolvimento relativo. Ciência Rural, 29(3):549-553.

Sakamoto, C. A. M., Lopes, W. D. Z., Buzzulini, C., Cruz, B. C., Felippelli, G., Lima, R. C. A. \& Costa, A. J. (2013). Anthelmintic efficacy of an oral formulation of Aurixazol against gastrointestinal nematodes of naturally and experimentally infected sheep. Veterinary Parasitology, 198(3-4):336-344.

Thomaz-Soccol, V. T., Souza, F. P., Sotomaior, C., Castro, E. A., Milczewski, V., Mocelin, G. \& Silva, M. C. E. (2004). Resistance of gastrointestinal nematodes to anthelmintics in sheep (Ovis aries). Brazilian Archives of Biology and Technology, 4741-47.

Vizard, A. L. \& Wallace, R. J. A. (1987). Simplified faecal egg count reduction test. Australian Veterinary Journal, 64(4):109-111.

Wood, I. B., Amaral, N. K., Bairden, K., Duncan, J. L., Kassai, T., Malone, J. B. \& Vercruysse, J. (1995). World Association for the Advancement of Veterinary Parasitology (W.A.A.V.P.) second edition of guidelines for evaluating the efficacy of anthelmintics in ruminants (bovine, ovine, caprine). Veterinary Parasitology, 58181213.

Recebido: 23 de setembro, 2019.

Aprovado: 13 de novembro, 2019.

Publicado: 9 de janeiro, 2020.

Licenciamento: Este artigo é publicado na modalidade Acesso Aberto sob a licença Creative Commons Atribuição 4.0 (CC-BY 4.0), a qual permite uso irrestrito, distribuição, reprodução em qualquer meio, desde que o autor e a fonte sejam devidamente creditados. 